\title{
Solitary subdural osteoma: A case report and literature review
}

\author{
LEI CAO ${ }^{1}$, LICHUAN HONG ${ }^{2}$, CHUZHONG LI $^{2}$, YAZHUO ZHANG ${ }^{2}$ and SONGBAI GUI ${ }^{1}$ \\ ${ }^{1}$ Department of Neurosurgery, Beijing Tiantan Hospital; ${ }^{2}$ Beijing Neurosurgical Institute, \\ Capital Medical University, Beijing 100050, P.R. China
}

Received April 1,2015; Accepted May 26, 2016

DOI: $10.3892 / 01.2016 .4736$

\begin{abstract}
Osteomas attached to the meninges unrelated to bone are extremely rare and their etiology has not been discussed previously in the English literature. Here, we report the case of a 54-year-old male patient with a right frontal subdural osteoma. The patient presented with a $~ 5$-month history of intermittent dizziness. Non-contrasted computerized tomography demonstrated a densely calcified mass attached to the inner surface of the right frontal skull. Magnetic resonance imaging (MRI) revealed this mass could be enhanced by contrast MRI. Intraoperatively, the hard mass was noted to be attached to the inner layer of the dura mater, and not associated with the bone. Histopathological examination revealed lamellated bony trabeculae lined by osteoblasts and the intertrabecular marrow spaces occupied by adipose tissue, which contributed to the MRI enhancement. It was speculated that subdural osteomas arose from ectopic osteoblasts derived from the embryological neural crest cells. The context of intertrabecular bone marrow contributed to the enhancement on MRI. Simple excision is the treatment of choice for symptomatic lesions.
\end{abstract}

\section{Introduction}

Osteomas are benign neoplasms characterized by the proliferation of compact, lamellar, mature, normal osseous tissue. The disease presents as an exophytic mass usually arising from the paranasal sinuses, facial bones, skull or mandible. Subsural osteomas attached to the meninges unrelated to bone are extremely rare (1-9). They are most likely located in the frontal region, according to the literature (1-9). The initiating factor that triggers the formation of subdural osteoma remains unclear; however, Choudhury et al (2), Aoki et al (3) and Sugimoto et al (4) have reported that head trauma may trigger the activation of dural ectopic osteoblasts. Symptomatic subdural osteomas may present as pressure symptoms

Correspondence to: Dr Songbai Gui, Department of Neurosurgery, Beijing Tiantan Hospital, Capital Medical University, 6 Tiantanxili, Dongcheng, Beijing 100050, P.R. China

E-mail: guisongbai@hotmail.com

Key words: subdural osteoma, dura mater, neural crest cell, oncology or epilepsy, and simple excision is the treatment of choice for symptomatic lesions. In this case report, we discuss the radiological and clinicopathological findings of a subdural osteoma in a 54-year-old male, and speculate on its etiological mechanism.

\section{Case report}

A 54-year-old male presented at Beijing Tiantan Hospital, Capital Medical University (Beijing, China) in June 2014, with a 5-month history of intermittent dizziness. He had a history of right zygomatic fracture 10 years prior without brain injury. Physical examination revealed no neurological abnormality. A non-contrast computerized tomography (CT) scan demonstrated a densely calcified mass beneath the right parietal bone, which exhibited a mild mass effect upon the right frontal lobe. Magnetic resonance imaging (MRI) revealed that the mass was iso- and hyperintense on T1-weighted imaging and iso- and hypointense on T2-weighted imaging. The mass and the attached dura mater could be enhanced by gadolinium-diethylenetriamine pentaacetic acid (Fig. 1). The tumor was misdiagnosed as calcified meningioma preoperatively, and a right frontal craniotomy was performed to remove it. The dura mater was observed to be intact intra-operatively, while the calcified mass attached to the inner layer of the meninges exhibited no association with the overlying cranial bone. The lesion was noted to be firm and it could be easily removed from the arachnoid membrane, so it was extracted en bloc without cortical injury. Pathological examination revealed lamellated bony trabeculae lined with osteoblasts, and the intertrabecular marrow spaces were occupied by adipose tissue having no active osteoblastic or osteoclastic activity (Fig. 2). The patient recovered uneventfully and was discharged without neurological deficits. The tumor did not recur during the 6-month follow-up period and the dizziness subsided.

Written informed consent was obtained from the patient prior to the publication of the present study.

\section{Discussion}

Subdural osteoma is recognized as a rare variant of intracranial osteoma and has an unclear etiology. A reactive mechanism may have been triggered by trauma in the present case, as certain authors have suggested previously (2-4). In the head, the most common anatomic sites are the frontal sinus, the ethmoidal sinuses and the mandible. However, subdural 


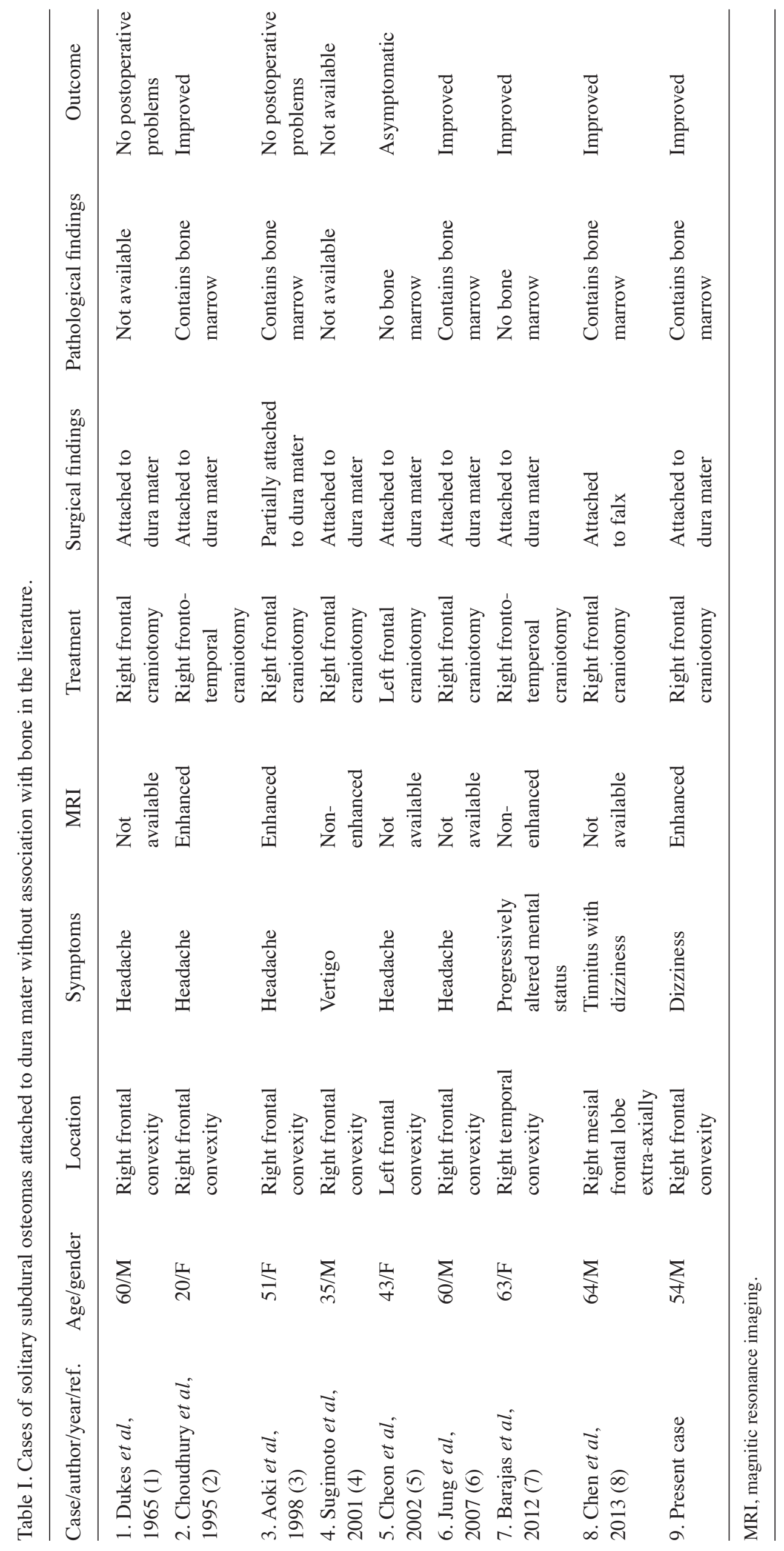



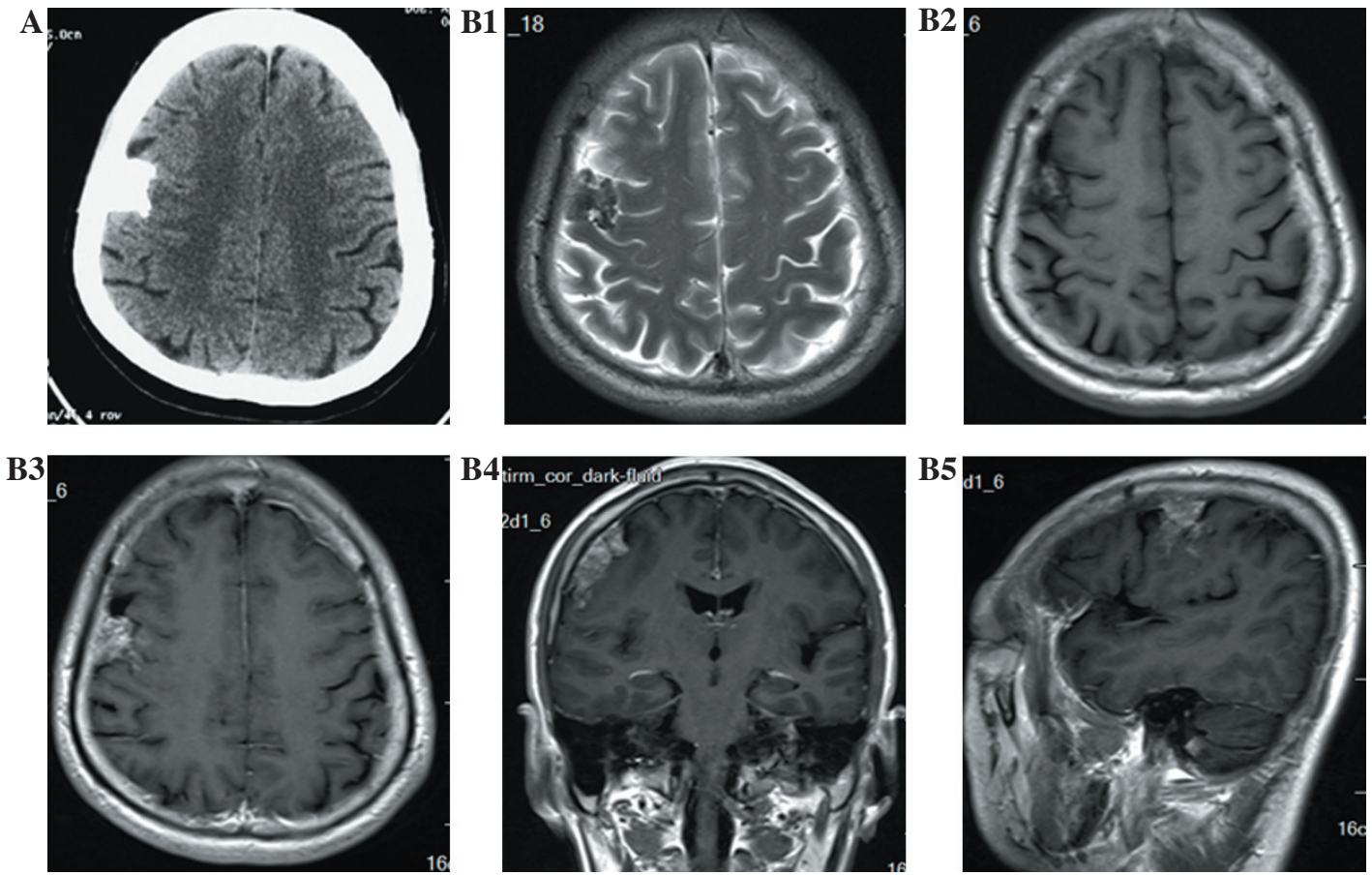

Figure 1. Appearance of the lesion on CT and MRI. (A) CT revealed a densely calcified mass attached to the inner surface of the right frontal skull. (B1-5) The mass was enhanced by (B1 and 2) non-contrast and (B3-5) contrast MRI. CT, computed tomography; MRI, magnetic resonance imaging.
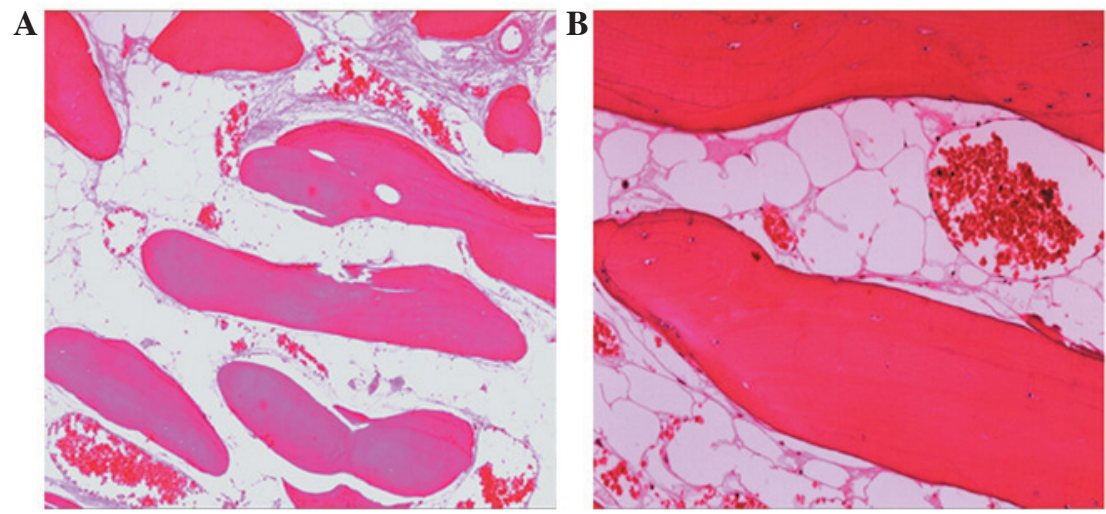

Figure 2. Histopathology of the resected specimen by hematoxylin and eosin staining. Lamellated bony trabeculae and the intertrabecular spaces are occupied by loose fibrovascular tissue (magnification A, x40; B, x200).

osteomas arising from the inner layer of the dura mater with no association with the covered bone are rarer. We reviewed the literature and noted that almost all previous cases of subdural osteomas were attached to the frontal dura or falx (Table I). It was located far more commonly on the right side than the left side (8:1 ratio). Fallon et al (9) also observed that intracranial meningeal osteomas were usually located at the convexity dura and falx junction around the superior sagittal sinus. The periosteum of the frontal bones and cells from the nasal septum, which are of neural crest origin, contribute to the falx cerebri and the adjacent dura (10). All of these predilection sites, including the frontal and ethmoidal sinuses and the mandible, are derived from the embryological neural crest cells. We speculated that the new bones arise from ectopic osteoblasts; this hypothesis requires further study.

This tumor type is mosts commonly associated with the symptoms of headache or dizziness, which may be due to the pressure on the underlying cerebral tissue. All patients in the cases studied presented with headache or dizziness, except one, who had a progressive, altered mental state (Table I).

On MRI, the appearance of the subdural osteomas was consistent with that of tumors. Table I reveals that three of the five subdural osteomas (cases 2, 3 and 9), containing intertrabecular bone marrow, were enhanced by contrast on MRI, while the enhanced MRI examination was not available for the other two cases (cases 6 and 8). Case 7, containing no bone marrow, was not enhanced. Therefore, the context of intertrabecular bone marrow contributed to the enhancement on MRI. Calcified meningioma often destroys surrounding bone as a consequence of extracranial extension (7), although it may also be enhanced on MRI. Subdural osteomas should be differentially diagnosed from meningeal ossifications, which are commonly multicentric and located on the dural-falx junction along both sides of the superior sagittal sinus (9). 
Simple excision is the treatment of choice for symptomatic lesions. These seldom recur following excision and they are not associated with malignant change.

\section{References}

1. Dukes HT and Odom GL: Discrete intradural osteoma. Report of a case. J Neurosurg 19: 251-253, 1962.

2. Choudhury AR, Haleem A and Tjan GT: Solitary intradural intracranial osteoma. Br J Neurosurg 9: 557-559, 1995.

3. Aoki H, Nakase H and Sakaki T: Subdural osteoma. Acta Neurochir (Wien) 140: 727-728, 1998

4. Sugimoto K, Nakahara I, Nishikawa M, Tanaka M, Terashima T, Yanagihara $\mathrm{H}$ and Hayashi J: Osteoma originating in the dura: a case report. No Shinkei Geka 29: 993-996, 2001 (In Japanese).
5. Cheon JE, Kim JE and Yang HJ: CT and pathologic findings of a case of subdural osteoma. Korean J Radiol 3: 211-213, 2002.

6. Jung TY, Jung S, Jin SG, Jin YH, Kim IY and Kang SS: Solitary intracranial subdural osteoma: intraoperative findings and primary anastomosis of an involved cortical vein. J Clin Neurosci 14: 468-470, 2007.

7. Barajas RF Jr, Perry A, Sughrue M, Aghi M and Cha S: Intracranial subdural osteoma: a rare benign tumor that can be differentiated from other calcified intracranial lesions utilizing MR imaging. J Neuroradiol 39: 263-266, 2012.

8. Chen SM, Chuang CC, Toh CH, Jung SM and Lui TN: Solitary intracranial osteoma with attachment to the falx: a case report. World J Surg Oncol 11: 221, 2013.

9. Fallon MD, Ellerbrake D and Teitelbaum SL: Meningeal osteomas and chronic renal failure. Hum Pathol 13: 449-453, 1982.

10. O'Rahilly R and Müller F: The meninges in human development. J Neuropathol Exp Neurol 45: 588-608, 1986. 